\title{
Off-the-person electrocardiography: performance assessment and clinical correlation
}

\author{
Hugo Plácido da Silva • Carlos Carreiras • \\ André Lourenço • Ana Fred · Rui César das Neves • \\ Rui Ferreira
}

Received: 17 February 2014 / Accepted: 10 December 2014 / Published online: 19 February 2015

(c) IUPESM and Springer-Verlag Berlin Heidelberg 2015

This work was partially funded by Fundação para a Ciência e Tecnologia (FCT) under grants PTDC/EEI-SII/2312/2012, SFRH/BD/65248/2009 and SFRH/PROTEC/49512/2009.

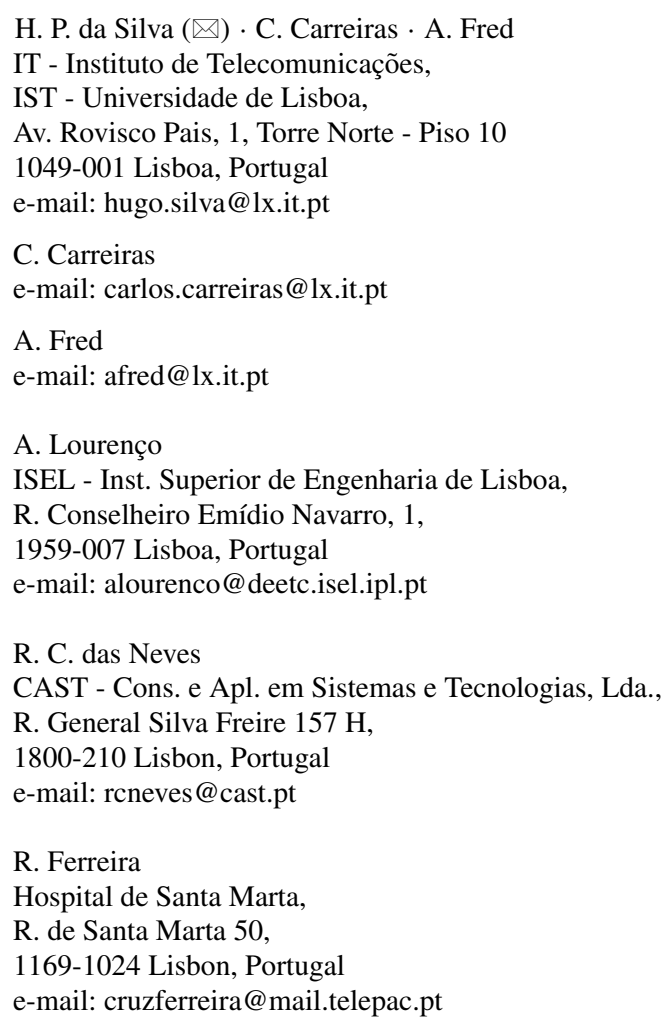

\begin{abstract}
Previous work by our group introduced a novel concept and sensor design for "off-the-person" ECG, for which evidence on how it compares against standard clinical-grade equipment has been largely missing. Our objectives with this work are to characterise the off-theperson approach in light of the current ECG systems landscape, and assess how the signals acquired using this
\end{abstract}

simplified setup compare with clinical-grade recordings. Empirical tests have been performed with real-world data collected from a population of 38 control subjects, to analyze the correlation between both approaches. Results show off-the-person data to be correlated with clinical-grade data, demonstrating the viability of this approach to potentially extend preventive medicine practices by enabling the integration of ECG monitoring into multiple dimensions of people's everyday lives.

Keywords Electrocardiography · Off-the-person . Taxonomy $\cdot$ Pervasive health

\section{Introduction}

Electrocardiography (ECG) is an established standard medical practice and a mainstream diagnostic technique. Although the first practical implementations of devices for human use can be dated back to 1887 [1], measurement methods are still mostly bound to hospital and short-time monitoring settings. With prevention being one of the main pilars for managing the risks associated with cardiovascular diseases management, new solutions with the potential to complement current practices, and accelerate early detection of abnormal conditions, can play a major role.

In [15] we introduced a sensor design for ECG data acquisition at the hand palms or fingers, further improved in [14], and capable of operating using non-gelled electrodes and conductive fabrics. This can greatly contribute for cardiovascular disesase management, especially in prevention and early detection, given that the sensor can be integrated in everyday use objects (e.g. a computer keyboard or a game station remote) rather than being attached to the body of the person, leading us to the concept of "off-the-person" ECG. 
Evidence regarding how this approach performs when compared to clinical-grade systems has been largely lacking. As such, this paper builds upon our previous work, by providing empirical evidence showing how our offthe-person approach correlates with data collected using a clinical-grade system. Given that segmentation is a fundamental step in computer-based ECG data analysis, we also assess the performance of several commonly used peak detection algorithms on off-the-person data.

It is important to stress that ECG signals are significantly different from the mere heart rate readings obtained using the systems incorporated in commonly found fitness equipment, both in terms of instrumentation requirements and information content. Our goal with the pursuit of this line of work is to contribute to further enrich current practices in cardiovascular disease prevention, by enabling ECG data acquisition to become more pervasive and seamlessly integrated in multiple aspects of peoples everyday lives.

The remainder of the paper is organized as follows: Section 2 provides a taxonomy for the current ECG devices landscape with respect to their level of practicality (or intrusiveness); Section 3 describes the methodology used for experimental data acquisition and analysis; Sections 4 and 5 present the comparative evaluation of the off-the-person approach against data collected using clinical-grade equipment; finally, Section 6 summarizes the main conclusions and future work.

\section{Taxonomy for the practicality of ECG devices}

Over the years, multiple ECG measurement approaches have been proposed. Voltage potential differential is the most common and widely used method, due to the bioelectrical phenomena underlying the cardiac activity [10]; other methods include capacitive and even ballistic sensing. In light of our line of thought, geared towards placing the sensors in objects with which the person interacts rather than directly on the body of the person, we found a top-level classification for the practicality of existing hardware devices with respect to their placement on the user to be missing. As such, in this paper we provide a taxonomy for which an overview is presented in Fig. 1.

\subsection{In-the-person}

This category defines equipment designed to be used directly inside the body of the person, which includes devices that are either surgically implanted, have subdermal application, or need to be ingested in pill-shaped form factors. These are generally used by chronic patients, and in extreme clinical scenarios for which less intrusive approaches do not suffice. Despite being internal to the body, most modern devices enable clinicians and even patients to access the data externally.

Implantable systems are one class of devices in this category. Artificial cardiac pacemakers have been around for decades, and are perhaps the best known example of implantable systems [17]. Modern devices for human use have extremely compact and lightweight form factors; a state-of-the-art example of such devices is Medtronic's recently announced Micra Transcatheter Pacing System ${ }^{1}$, which has $24 \mathrm{~mm}$ in length and a volume of less than $1 \mathrm{~cm}^{3}$.

Another type of devices belonging to this class can be defined as Minimally Invasive, including systems such as Implantable Loop Recorders (ILR) and pill-like systems. Despite the fact that these devices are designed for use inside the body, they either have subdermal application through simple medical procedures or are administrated orally. ILR's are a typical example of a minimally invasive system, in which the application is done through a small incision that only requires local anesthesia; once the device is applied, it enables the continuous logging of the cardiac activity.

\subsection{On-the-person}

Most of the devices used nowadays in clinical and wellbeing applications can be classified in this category, which defines ECG measurement methods that require the use of a device or some of its components directly attached to the body surface (e.g. the electrode leads). Examples of such devices range from bedside monitors or medical diagnostics systems used in a hospital setting, to personal devices for self-monitoring of 1-lead ECG data, such as chest straps or attachable patches for heart rate monitoring (e.g. Zephyr BioHarness \& BioPatch ${ }^{2}$ ).

Standard ECG devices such as workbench, cart or bedside units can be defined as Stationary systems. These devices generally use up to 12 leads mounted on the chest and limbs, and require the use of conductive gel or pastes. Also, devices in this class are mostly designed to be used within a limited physical space to which the person is bound to, this being a medical care facility, an ambulance, an ambient assisted living unit, or other environments alike.

Over the years, a vast effort has been made in the field of Ambulatory systems. This class includes devices that range from Holter monitors used for near-continuous cardiac data logging, typically over the course of $24 \mathrm{~h}$ periods, to t-shirts and other wearable form factors. Representative examples of these devices are the VitalJacket from BioDevices ${ }^{3}$ [5],

\footnotetext{
${ }^{1}$ http://newsroom.medtronic.com/phoenix.zhtml?c=251324\& $\mathrm{p}=$ irol-newsArticle \&id=1883208

${ }^{2} \mathrm{http}: / / \mathrm{www} . z e p h y r a n y w h e r e . c o m /$ products/

${ }^{3}$ http://www.biodevices.pt
} 
Fig. 1 Overview of the intrusiveness and potential outreach of ECG data acquisition systems

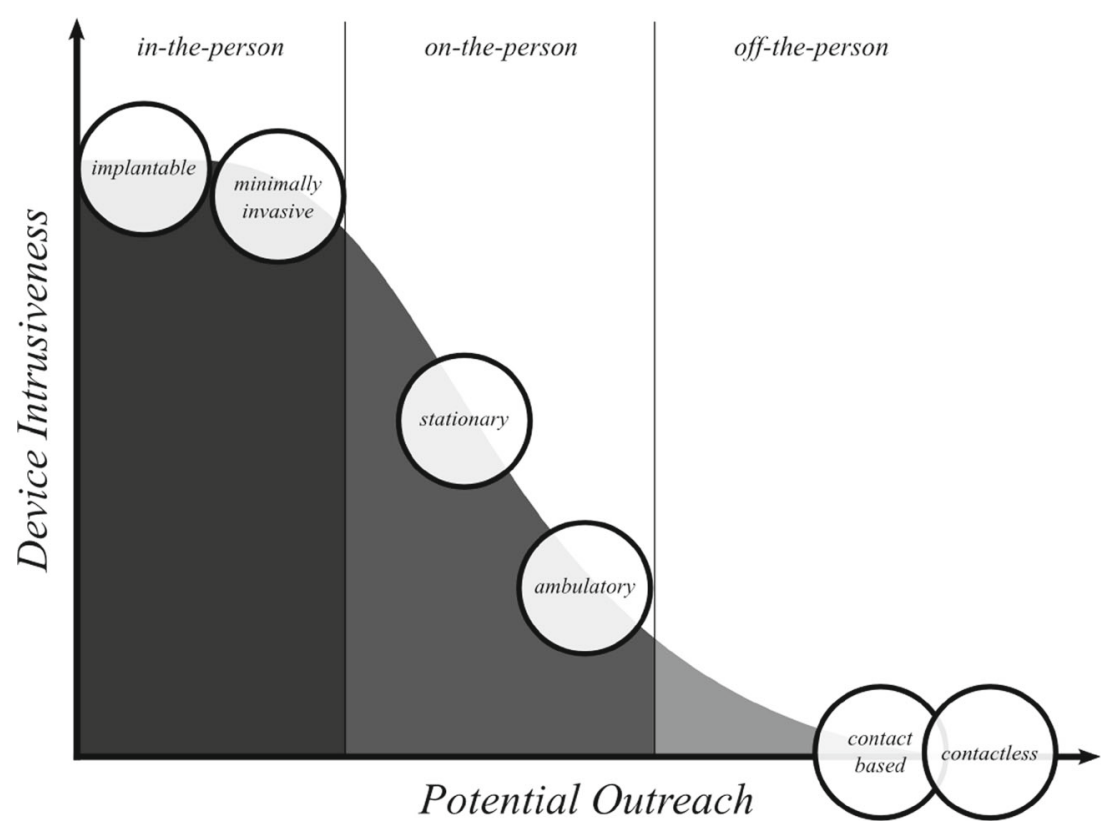

and the AAL+ necklace described in [16] that uses dry $\mathrm{Ag} / \mathrm{AgCl}$ electrodes and enables heart rate measurement on the user's neck.

Within the ecosystem of on-the-person wearable devices, an increasingly growing trend has been seen within the current state-of-the-art towards integrating ECG sensors into mobile phones or bracelets. Examples of such trend are the Samsung SIMBAND ${ }^{4}$, the AliveCor ${ }^{5}$ smartphone accessory, and the EPI Life ${ }^{6}$ purpose-built phone fitted with ECG sensors.

\subsection{Off-the-person}

In a pervasive healthcare framework, one can easily envision several use cases where the sensors do not need to be with the person, but instead are embedded into everyday use objects; this brings us to the concept of off-the-person ECG. A major advantage of this approach is the fact that the sensor placement does not require a voluntary action from the user, unlike, for example, wearable on-the-person devices. These novel approaches are actually well aligned with the future trends envisioned in terms of medical applications of technology [18].

One category in this space comprises Contact Based systems, that is, devices that still require the use of elec-

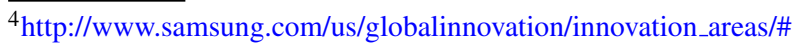
simband

${ }^{5}$ http://www.alivecor.com

${ }^{6} \mathrm{http}: / /$ heartronics.com.my
}

trodes in contact with the body of the person, but in which design considerations were taken to allow their integration in objects with which the person regularly interacts, without impacting the normal routines. An example of contact based off-the-person sensing can be found in [15], where a sensor designed for use with dry electrodes was proposed, targeting ECG data acquisition at the hand palms or fingers. Although primarily created to perform measurements at the chest, the previously mentioned AliveCor smartphone accessory can also be seen as another example of an off-theperson device, given that the ECG measurements can also be performed by letting the phone rest on the fingers. This type of sensors can be easily embedded into items such as a computer keyboard, a gaming station controller, among many other items, enabling their use for electrocardiography in a pervasive health framework, and promoting novel periodic and long-term monitoring paradigms.

Another class is Contactless systems, which includes both capacitive and mechanical sensing. It is important to highlight that these approaches are not capable of measuring the tradicional ECG signal, but they do measure an ECG-like activity, with the advantage of relieving the constraints associated with needing to have the electrodes in contact with the body of the person. Capacitive methods work by measuring electric field changes induced by the bioelectric activity of the heart $[3,11,13]$, and can be designed to measure the ECG at distances of $\sim 1 \mathrm{~cm}$ or more, even with clothing in-between the body and the sensor. Mechanical methods measure heart-related events by sensing the vibrations propagated to the body surface after the contraction of the cardiac musculature. This is known as ballistocardiography or seismocardiography, and current 
approaches have been based on the use of accelerometers or electromechanical films [12].

\section{Experimental procedures}

A long-standing question with respect to our proposed approach for off-the-person ECG has been the relation between the signals obtained with this type of sensor, and those obtained with clinical-grade stationary on-the-person devices, which are an ECG gold standard. It is important to highlight that on our approach, the hand palms sensor placement and the use of non-gelled electrodes relief important pre-established constrains in gold standard methodologies $[14,15]$. Therefore, the goal of this comparison is not to prove or demonstrate any physiological principles, but instead, to empirically analyse if our signals correlate with one (or more) leads of what is known to be the gold standard, thus validating its potential use in real-world applications.

\subsection{Data acquisition}

To address this question, we have conducted experimental tests involving 38 volunteers (50\% females), in which simultaneous recordings were performed using a Philips PageWriter Trim III series ECG device (hereinafter referred to as Philips), and our off-the-person sensor design as presented in [14] (hereinafter referred to as BITalino). Figure 2 provides an overview of the experimental setup used in our work.

The Philips device was used in the standard 12-lead setting (I-III, V1-V6, aVF, aVL, aVR), with conductive paste applied to each of the electrodes, while our sensor was used in the virtual ground setting with dry $\mathrm{Ag} / \mathrm{AgCl}$ electrodes; for data acquisition we used our sensor with the BITalino system [14]. Subjects were asked to hold one of the electrode leads in the right hand and the other on the left hand. Data collection was performed using the standard clinical procedure, in which the subject is lying down, at rest, in an examination bed. The BITalino system acquired data continuously. Given that the Philips device only records 10 seconds of data per run, we acquired three sequential runs, thus amounting to 30 seconds of data per subject per session. Ground truth data annotation for R-peak labelling was performed manually by a human expert that reviewed all of the records.

This setup with the users at rest, was motivated by the fact that our approach already relaxes several requirements of gold standard approaches, reason for which we left the analysis of dynamic scenarios to be the focus of future work. Still, it is important to stress that there are multiple realworld situations where users are inherently at rest, with

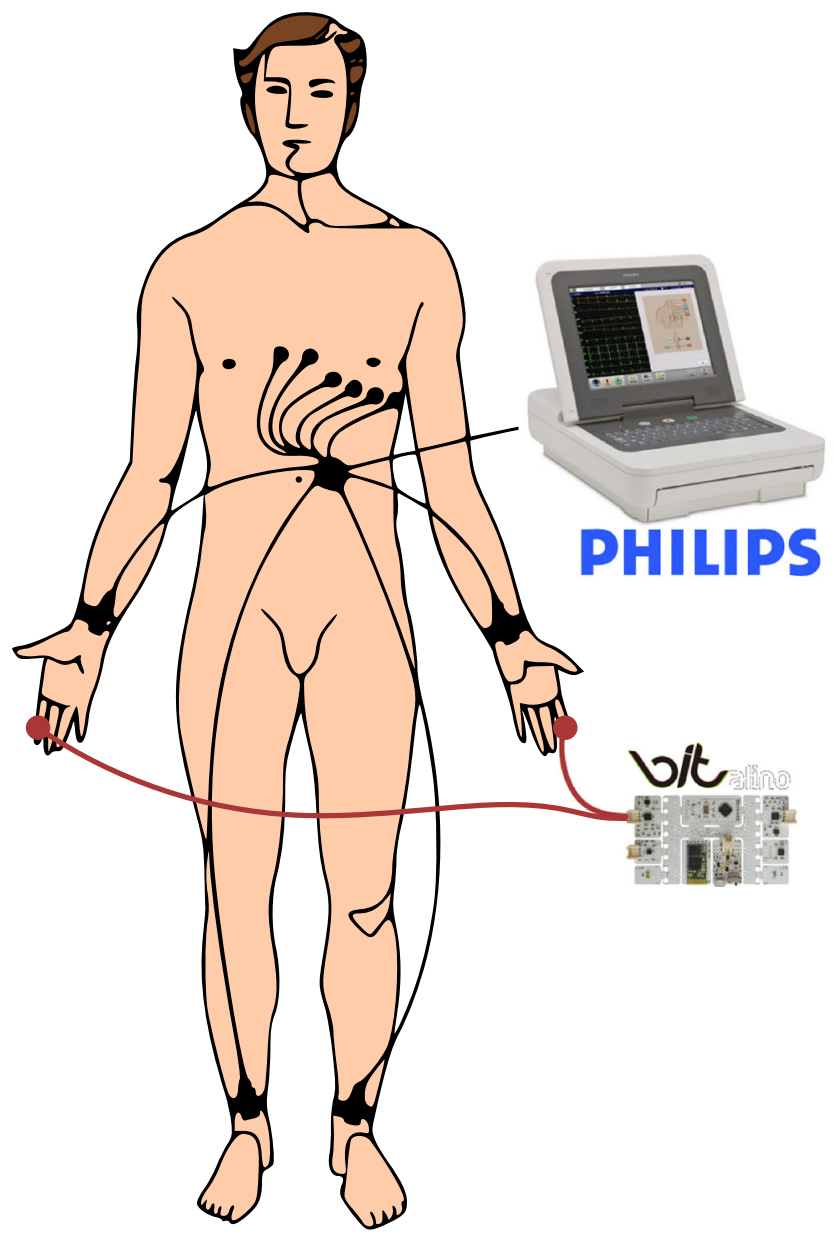

Fig. 2 Experimental setup used in our work, involving a Philips PageWriter Trim III classical 12-lead ECG placement system, along with our off-the-person sensor approach using dry electrodes placed at the hands (drawings not to scale)

limited or no dynamic activity of locations such as the hand palms. Examples of such use cases include working with a computer keyboard or using a remote control of a gaming station, just to name a few.

\subsection{Preprocessing}

Given that the Philips PageWriter device has a sampling rate of $500 \mathrm{~Hz}$, whereas in BITalino we used $1000 \mathrm{~Hz}$, the raw data from our device was post-processed in order to perform a downsampling to $500 \mathrm{~Hz}$, after filtering the signal with a butterworth digital filter, of order 3 , in the frequency band $[0.5,40] \mathrm{Hz}$. Another core aspect that we have considered in our analysis is the fact that data acquisition is being done by two independent systems.

The Philips device does not provide a direct approach to synchronization at the hardware level; to overcome this problem, in the post-processing we also performed the temporal alignment of both signals by first normalizing the DC 


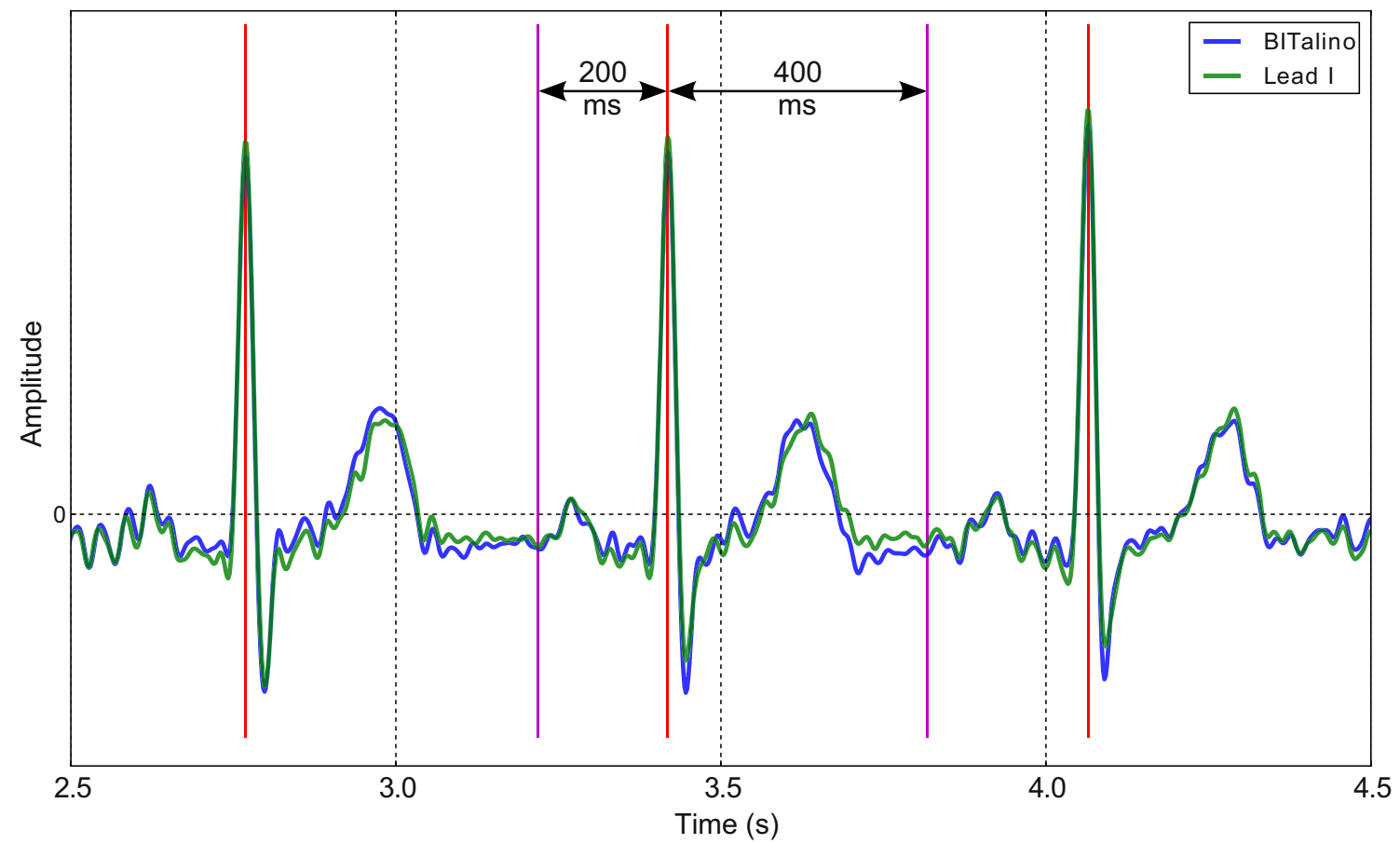

Fig. 3 Synchronized BITalino and Philips Lead I signals for subject S8, and example of R-peak detection for segmentation using the best and the positions of the $\mathrm{R}$ peaks are plotted in red vertical lines, while performing algorithm [9]; normalization was performed using Eq. 1, the limits of one of the heartbeats are shown in magenta

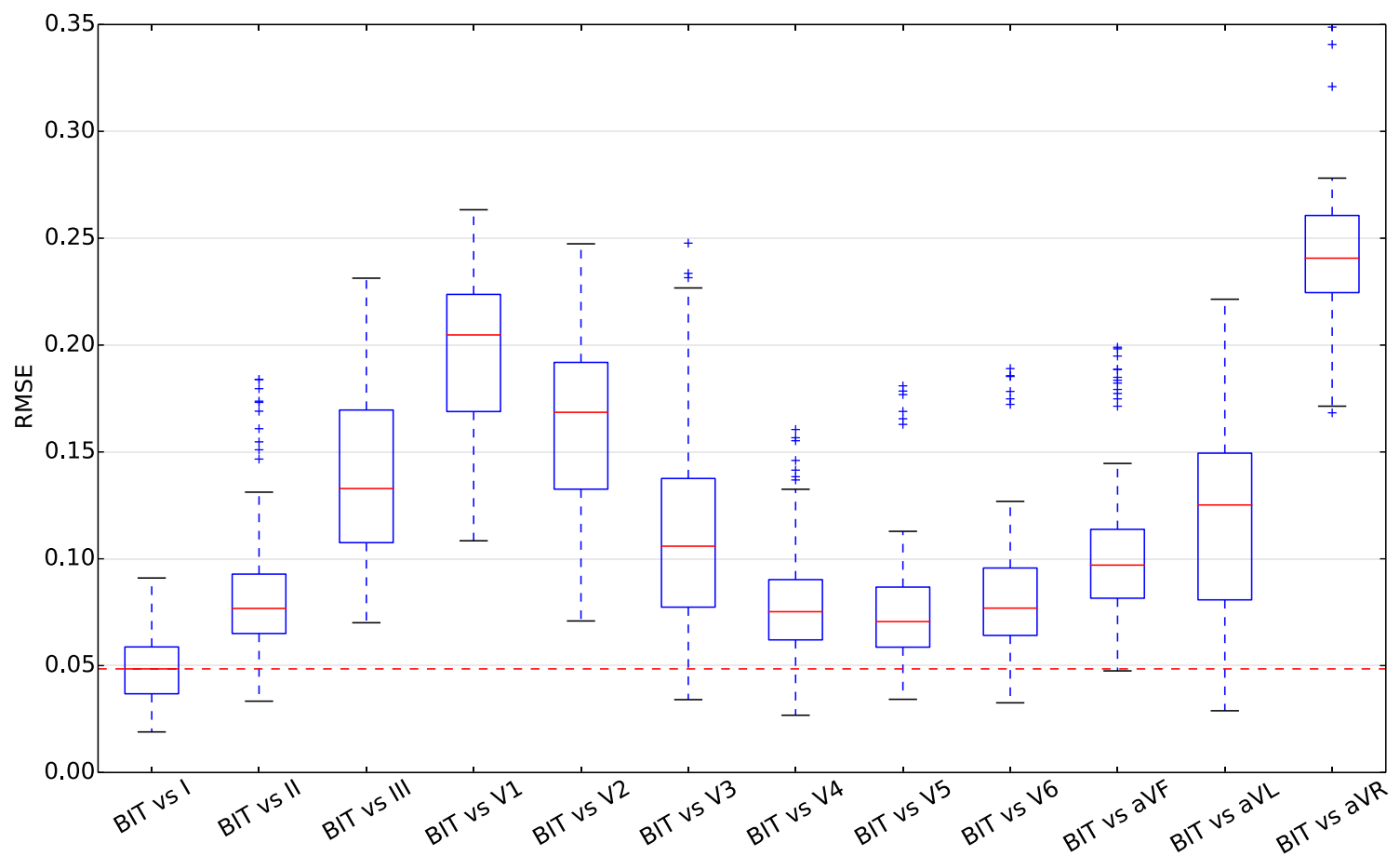

Fig. 4 Root Mean Square Error between the BITalino and Philips Lead I synchronized signals for all subjects; values are the averages across all runs 
offset and amplitude of the signals as shown in Eq. 1, where the original signal $x[k]$ is subtracted of its mean $\mu$, and then divided by the total amplitude variation.

$x_{\text {norm }}[k]=\frac{x[k]-\mu}{\max _{k}\{x[k]\}-\min _{k}\{x[k]\}}$

We then aligned the signals between corresponding BITalino and Philips time series, by computing the time delay for which the cross-correlation between them is maximum (2). Note that we use the Lead I from the Philips system to determine the delay, given that, as we will demonstrate throughout the following sections, this derivation is the one that better correlates with the off-the-person data.

$d^{*}=\arg \max _{d \in \mathbb{Z}} \sum_{k=-\infty}^{+\infty} x[k] y[k+d]$

Figure 3 shows an example of the synchronized signals for one of the acquisition runs, where we can observe that there is an almost exact match between the two signals. This is further validated by computing the Root Mean Square Error (RMSE), as defined in Eq. 3.

$\operatorname{RMSE}(x, y)=\sqrt{\frac{\sum_{k=1}^{n}(x[k]-y[k])^{2}}{n}}$

In Fig. 4 we present the RMSE between the synchronized BITalino signal and each of the Philips leads, for the entire database. It is important to highlight that for this analysis, the signals were preprocessed as described in the beginning of Section 5. We can see that the error to Lead I is indeed small $(<0.05)$, although we lack a reference with which to compare it to.

\section{Segmentation performance}

An important step in ECG data analysis is the identification of fiducial points within the signal trace, the R-peak generally being the reference complex in the segmentation

Table 1 Total number of detected R-peaks across all records

\begin{tabular}{llll}
\hline Algorithm & Total & Philips & BITalino \\
\hline Eng. \& Zee.[9] & & 1691 & 1692 \\
Christov[4] & 1712 & 1927 & 1931 \\
Hamilton[8] & & 2380 & 2250 \\
Zong et al.[19] & & 1594 & 1568 \\
\hline
\end{tabular}

process. As such, we sought to evaluate if there are noticeable differences in the peak detection performance when using off-the-person data. We chose four R-peak detection algorithms.

The detectors by Engelse and Zeelenberg [6], Hamilton [8], and Christov [4], were selected due to the fact that they are widely found in reference literature. Zong et al. [19] has a simpler implementation, thus potentially being more sensitive to noisy data, reason for which we also included it in our comparison. In addition to the previous references, we refer the reader to the work by [2,9] and references therein for additional details on each of these algorithms.

For our segmentation tests, the collected data from both devices was pre-processed using a bandpass FIR filter with a $300 \mathrm{~ms}$ window and a passing band spaning the $[5,20] \mathrm{Hz}$ range. From the filtered data, we compared the number of R-peaks detected (Table 1), as well as the precision.

Precision, as described in Eq. 4, is the ratio between the number of R-peaks correctly detected from the off-theperson data when validated against the ground truth data $(T P)$, and the total number of detected R-peaks from the off-the-person data $(F P+T P ; F P$ being the total number of incorrectly detected R-peaks). We use the manually labelled R-peaks as ground truth (or reference) to benchmark against. A given R-peak detected in the off-the-person data is considered to be correct (that is, a $T P$ ), if it differs by $<50$ milliseconds from a potential counterpart in the clinical-grade data, and it is considered incorrect otherwise (that is, a $F P$ ).

$\operatorname{Precision}(\%)=\frac{T P}{F P+T P} \times 100$

The results for R-peak detection precision are presented in Table 2. We used each algorithm for R-peak detection on the clinical-grade data and on off-the-person data. Figure 3 shows an excerpt of a signal annotated with the R-peaks as detected using the best performing algorithm. For segmentation, we consider the signal segment corresponding to $200 \mathrm{~ms}$ before and $400 \mathrm{~ms}$ after each R peak, amounting to a total of $600 \mathrm{~ms}$, which corresponds to the RR interval with a heart rate of $100 \mathrm{bpm}$.

Table 2 Average R-peak detection precision of commonly used peak detectors on off-the-person and clinical-grade data $(\mu \pm \sigma \%)$

\begin{tabular}{lll}
\hline Algorithm & Philips & BITalino \\
\hline Eng. \& Zee.[9] & $99.00 \pm 4.58$ & $98.36 \pm 7.15$ \\
Christov[4] & $95.03 \pm 18.93$ & $94.86 \pm 18.95$ \\
Hamilton[8] & $92.62 \pm 18.89$ & $91.54 \pm 20.37$ \\
Zong et al.[19] & $90.32 \pm 19.37$ & $90.27 \pm 18.77$ \\
\hline
\end{tabular}


Our analysis has revealed that, on average, R-peak detection from off-the-person ECG data has a precision above $90 \%$ for all algorithms, the best performing algorithm being the detector by Engelse \& Zeelenberg [9] with > 98\% precision. Furthermore, results show the segmentation performance to be fairly comparable to that achieved when using clinical-grade data.

\section{Correlation of clinical and off-the-person data}

With segmentation results pointing towards a comparable performance, we set out to perform a more granular assessment, by evaluating how the actual morphology of each individual heartbeat waveform compares between the signals collected using our off-the-person approach and the clinical-grade equipment.

Given that the analog signal conditioning circuitry is different in both devices, the raw data collected from each device was passed through a filtering preprocessing step. Our sensor uses the most restrictive frequency bandwidth, which spans the $[0.5,40] \mathrm{Hz}$ range; as such, we designed a digital filter with a high pass cutoff frequency of $0.5 \mathrm{~Hz}$ and a lowpass cutoff frequency of $40 \mathrm{~Hz}$, and filtered both signals using the same procedure, which consisted on a $3^{r d}$ order zero-phase forward and reverse Butterworth filter [7].

All the individual heartbeat waveforms were segmented using the best performing algorithm, that is, the Engelse \& Zeelenberg approach [9]. Our focus in this part of the study is on determining the morphological similarity between individual heartbeat waveforms collected using each of the sensor devices. Considering that dry electrodes are used in our sensor, and also due to the fact that the clinicalgrade equipment uses leads scattered through different anatomical locations, the magnitude of the signals collected by each device is affected by a variable scale factor. To account for such differences, in this study we adopted the cosine similarity (5); this metric is quite convenient given that it provides a magnitude-independent result normalized to $[0,1]$.

$$
D_{\cos }(x, y)=1-\frac{\sum_{k=1}^{n} x[k] y[k]}{\sqrt{\sum_{k=1}^{n} x[k]^{2} \sum_{k=1}^{n} y[k]^{2}}}
$$

The boxplot in Fig. 5 characterises the distances between the off-the-person and the medical-grade leads. Results
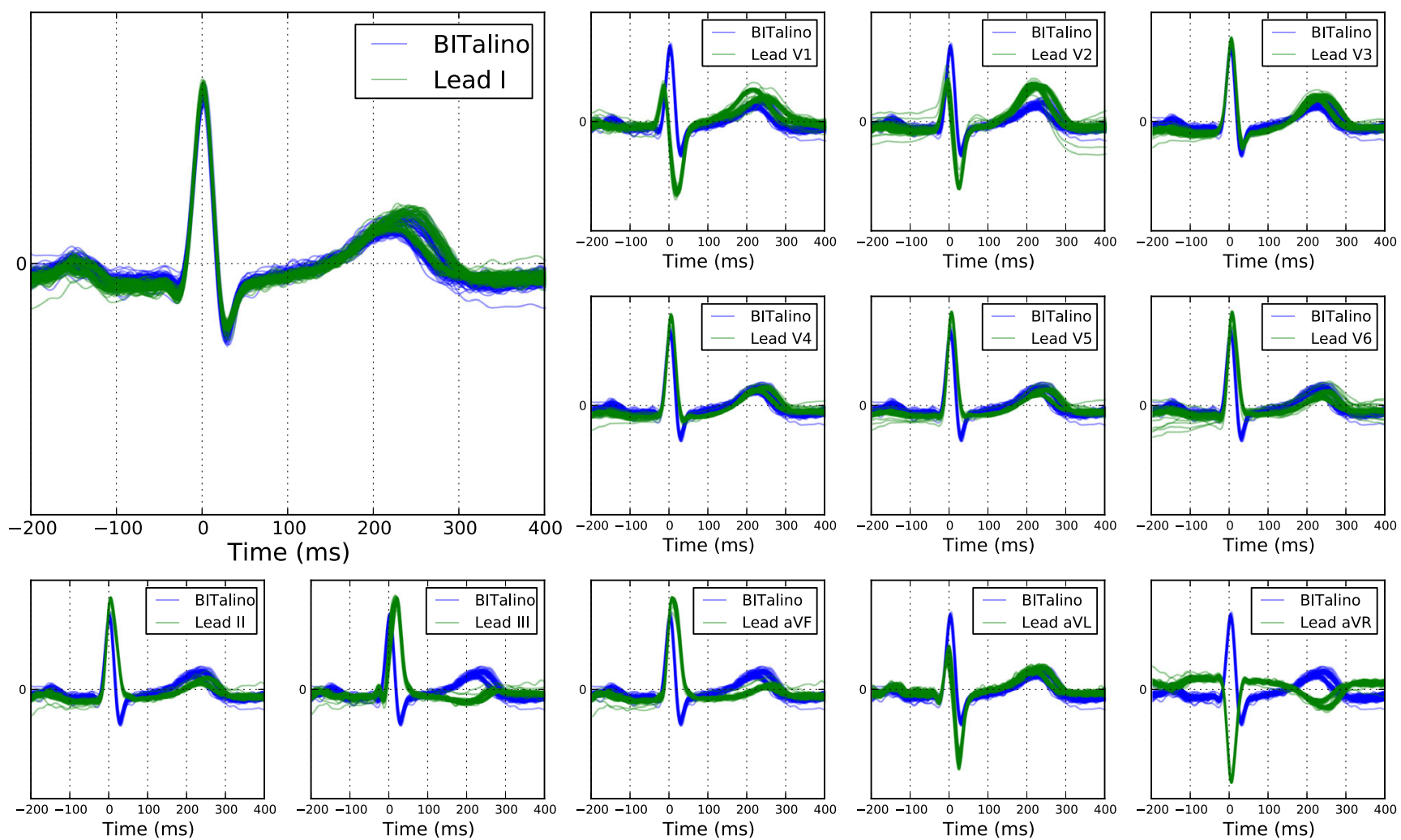

Fig. 5 Boxplot of the distance between the off-the-person and each of the medical-grade leads 
316

Health Technol. (2014) 4:309-318

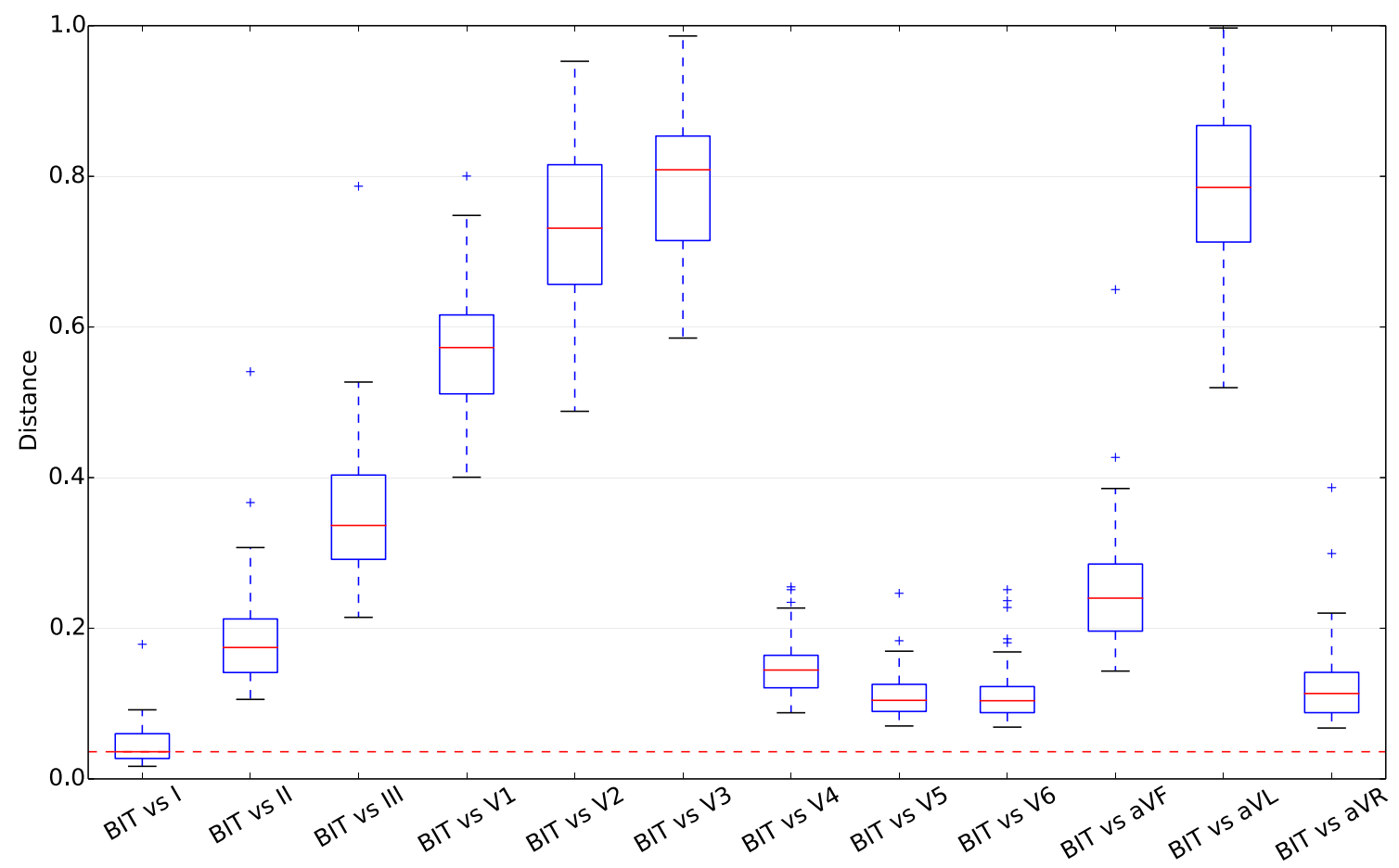

Fig. 6 Example of the segmented heartbeat waveforms obtained with the off-the-person and each of the clinical-grade leads for subject S8

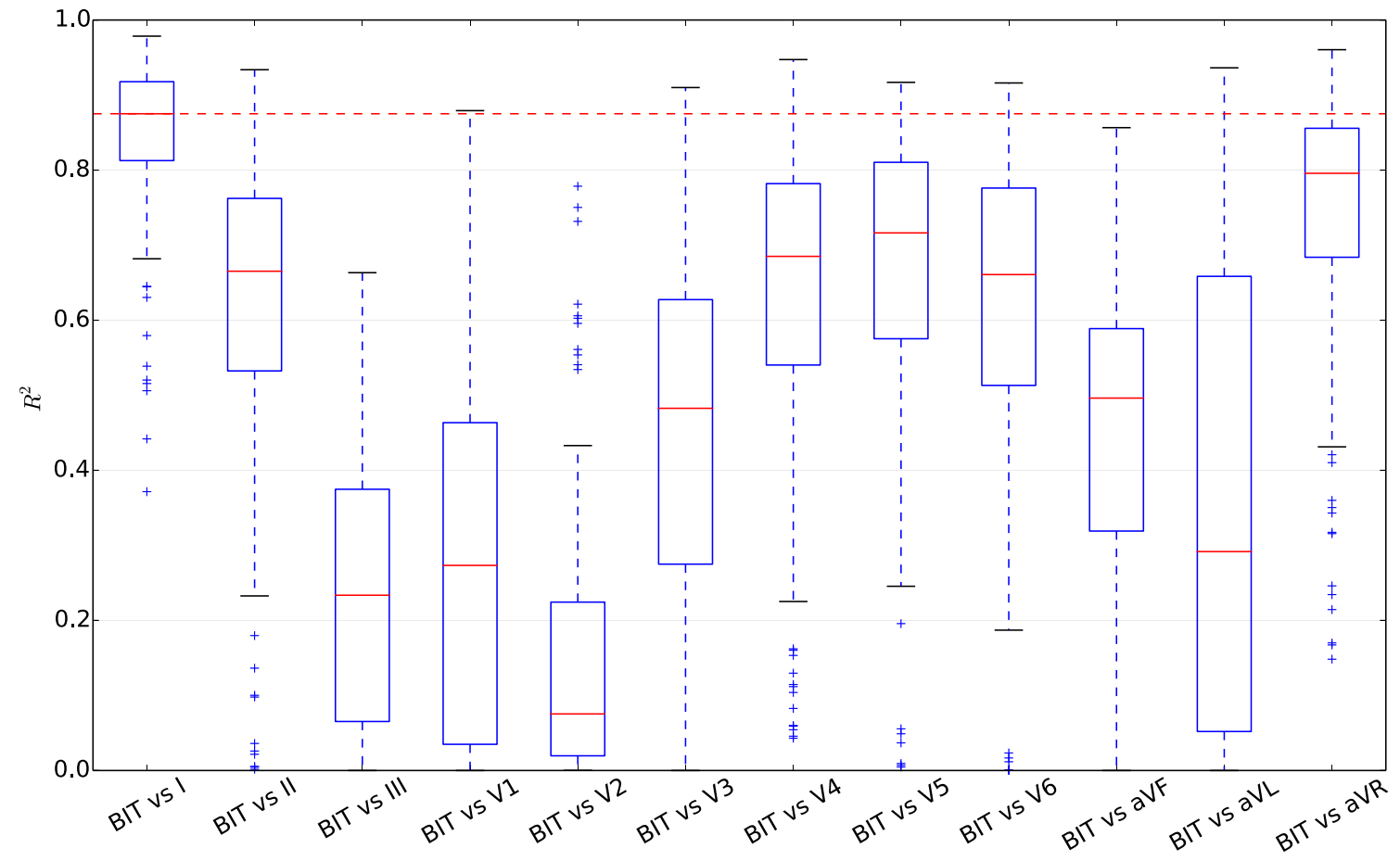

Fig. $7 \mathrm{R}^{2}$ coefficient of determination computed between the off-the-person and each of the medical-grade leads

Springer 
show that, in the overall, data collected at the hand palms or fingers using our approach correlates best with the clinicalgrade Lead I. For a better visual reference, Fig. 6 depicts an overlay with the segmented individual heartbeat waveforms for one of the tested subjects. The off-the-person data is represented in blue, while the clinical-grade data for each of the leads is represented in green; in this case, the off-the-person lead and the Lead I data closely match.

$$
\begin{aligned}
& S S_{x x}=\sum_{k=1}^{n} x[k]^{2}-n \mu_{x}^{2} \\
& S S_{y y}=\sum_{k=1}^{n} y[k]^{2}-n \mu_{y}^{2} \\
& S S_{x y}=\sum_{k=1}^{n} x[k] y[k]-n \mu_{x} \mu_{y} \\
& R^{2}=\frac{S S_{x y}^{2}}{S S_{x x} S S_{y y}}
\end{aligned}
$$

The median cosine distance between the off-the-person waveform and the Lead I waveform of the clinical-grade device sits below 0.04 , and low variability across the overall set of subjects was found. The coefficient of determination $R^{2}(9)$ is characterised by the boxplot shown in Fig. 7, the median value being 0.875 , which allows us to conclude that both signals are fairly correlated.

Given that each of the leads is in contact with a different limb, unlike local differential setups, there is a large enough potential difference to be more immune to EMG contribution. Still, if the user grasps the sensor lead vigorously, the signal-to-noise ratio will degrade. Furthermore, due to the use of non-gelled electrodes, mechanical action in the skin/electrode interface (e.g. sliding the hand on the electrode) will introduce momentary artifacts in the signals. A more in-depth study of these issues is the focus of ongoing and future work.

Nonetheless, this approach is particularly interesting as it can enable more periodic cardiovascular assessments in the convenience of the users' everyday life. If one has a computer keyboard fitted with a sensor, one can be followed-up virtually every day (unlike current practices in which there is the need to visit a clinical facility), thus enriching the current practices in what concerns prevention, early detection, and management of cardiovascular diseases. It is also important to further reinforce the fact that ECG measurements obtained with our approach, are significantly different from the simple heart rate readings provided by fitness equipment.

\section{Conclusions and future work}

In previous work, our team has devised a sensor design capable of measuring ECG data at the hand palms or fingers using dry electrodes. As depicted in Fig. 8, our approach enables novel use cases in which the sensor device is fitted into a multitude of objects such as a computer keyboard, a game station remote controller, and others alike. We build upon the idea that, in many cases, the sensors do not need to be permanently applied to the body of the users, but may instead be embedded into objects with which they regularly interact. In particular, we have focused on Electrocardiography (ECG), leading us to the concept of off-the-person ECG, which relaxes several requirements of conventional

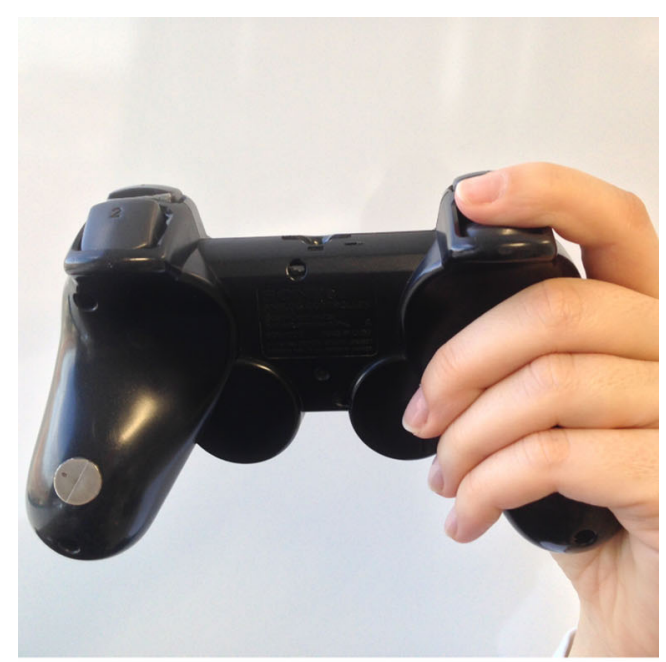

(a) Remote control of a gaming station

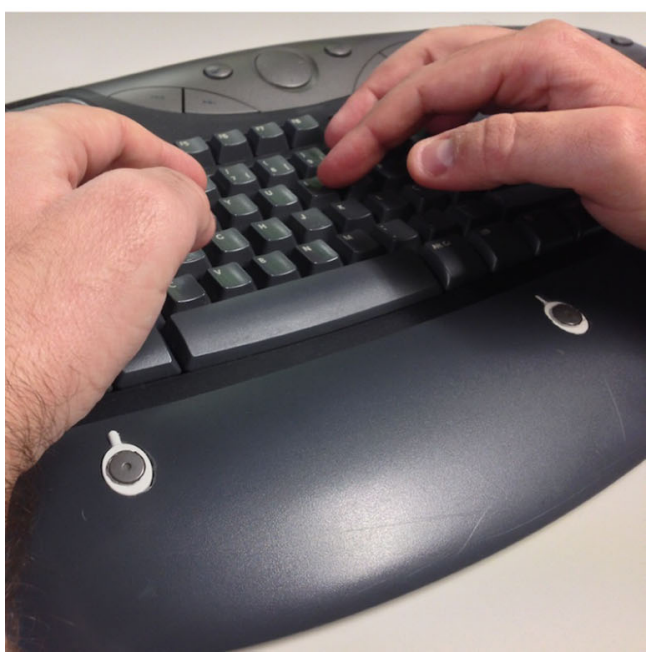

(b) Computer keyboard

Fig. 8 Examples of a contact based off-the-person ECG sensor integrated into everyday use objects 
approaches (e.g. pre-gelled electrodes, cable placement at the chest, among others).

Although we have proven the technical accuracy of our sensor design, an outstanding question for which evidence has been mostly lacking regards the relation between the data collected using our off-the-person approach and data collected with gold standard equipment. In this paper we presented a taxonomy that defines ECG data acquisition methods with respect to their practicality level, and provided experimental evidence that points towards a fair correlation between off-the-person and clinical-grade data. Comparative tests have been made with real-world empirical data to assess the segmentation performance and morphological waveform correlation, with results showing both a high precision in R-peak detection for segmentation, and a strong correlation between individual waveforms segmented from synchronized off-the-person and clinical-grade data.

Future work will focus on further validating our approach by benchmarking the system in dynamic scenarios, increasing the control population, and developing clinical studies with pathological populations, to characterise the type of conditions that can be detected using our approach, as well as the feasibility of our approach for the early detection and management of cardiovascular diseases.

Acknowledgements The team would like to thank all the anonymous volunteers that willingly agreed to participate in this sudy and also to the team that supported the data acquisition process at the Centro Hospitalar Lisboa Central, in particular to the head of pneumology technicians Isabel Carlos.

Conflict of interests The authors declare that they have no conflict of interest.

\section{References}

1. Besterman E, Creese R. Waller - pioneer of electrocardiography. Br Heart J. 1979;42:61-4.

2. Canento F, Lourenço A, Silva H, Fred A, Raposo N. On real time ECG algorithms for biometric applications. Proceedings of the 6th BIOSIGNALS Conf.; 2013. p. 228-35.
3. Chi Y, Jung TP, Cauwenberghs G. Dry-contact and noncontact biopotential electrodes: methodological review. IEEE Rev Biomed Eng. 2010;3:106-19.

4. Christov II. Real time electrocardiogram QRS detection using combined adaptive threshold. Biomed Eng Online. 2004;3(1).

5. Cunha J, Cunha B, Xavier W, Ferreira N, Pereira A. VitalJacket: a wearable wireless vital signs monitor for patients' mobility. Proceedings of the Avantex Symposium; 2007.

6. Engelse WAH, Zeelenberg C. A single scan algorithm for QRSdetection and feature extraction. Comput Cardiol. 1979;6:37-42.

7. Gustafsson F. Determining the initial states in forward-backward filtering. IEEE Trans Sig Process. 1996;44(4):988-92.

8. Hamilton P. Open source ECG analysis. Computers in Cardiology. 2002.

9. Lourenço A, Silva H, Leite P, Lourenço R, Fred A. Real time electrocardiogram segmentation for finger based, ECG biometric. Proceedings of the 5th BIOSIGNALS Conference; 2012. p. 49-54.

10. Malmivuo J, Plonsey R. Bioelectromagnetism: principles and applications of bioelectric and biomagnetic fields. New York: Oxford University Press; 1995.

11. Martins R, Primor D, Paiva T. High-Performance groundless EEG/ECG capacitive electrodes. Proceedings of the 6th IEEE MeMeA; 2011. p. 503-6.

12. Postolache OA, Girao PMBS, Mendes J, Pinheiro EC, Postolache G. Physiological parameters measurement based on wheelchair embedded sensors and advanced signal processing. IEEE Trans Instrum Meas. 2010;59(10):2564-74.

13. Prance RJ, Debray A, Clark TD, Prance H, Nock M, Harland CJ, Clippingdale AJ. An ultra-low-noise electrical-potential probe for human-body scanning. Meas Sci Technol. 2000;11:291-97.

14. Silva H, Guerreiro J, Lourenço A, Fred A, Martins R. BITalino: A novel hardware framework for physiological computing. Proceedings of the Int'l Conf. on Physiological Computing Systems (PhyCS); 2014. p. 246-53.

15. Silva H, Lourenço A, Lourenço R, Leite P, Coutinho D, Fred A. Study and evaluation of a single differential sensor design based on electro-textile electrodes for ECG biometrics applications. Proceedings of the 10th IEEE Sensors Conference; 2011. p. $1764-67$.

16. Silva H, Palma S, Gamboa H. AAL+: Continuous institutional and home care through wireless biosignal monitoring systems. In: Handbook of Digital Homecare, vol. 3. Berlin: Springer; 2011, pp. 115-42.

17. Timperley J, Leeson P, Mitchell A, Betts T. Cardiac Pacemakers and ICDs (Oxford Specialist Handbooks in Cardiology). Oxford: Oxford University Press; 2008.

18. Topol E. The Creative Destruction of Medicine. New York: Basic Books; 2012.

19. Zong W, Heldt T, Moody G, Mark R. An open-source algorithm to detect onset of arterial blood pressure pulses. Comput Cardiol. 2003;30:259-62. 